\title{
Myocardial perfusion SPECT "screening" at the start of dialysis
}

\author{
Sabha Bhatti, $M D{ }^{a}$ and Abdul Hakeem, $M D^{b}$
}

See related article, pp. 438-447

End stage renal disease (ESRD), defined by the need for renal replacement therapy (dialysis or renal transplantation), is a major health problem with an estimated prevalence in the US of half a million people and an annual incidence of $>110,000$ new dialysis beneficiaries in 2008 . The annual economic cost is estimated at around 39 billion dollars. In 2007, mortality rates for those who were receiving dialysis were 6.7 to 8.5 times greater than those of the general US population. ${ }^{1}$ In a recent European study involving $>123,000$ patients starting dialysis, age standardized cardiovascular mortality was 8.8 times and noncardiovascular mortality were 8.1 times higher than in the general population. ${ }^{2}$ Patients starting dialysis are hence a high-risk group in need for further risk stratification.

According to the National kidney foundation guidelines for evaluation of CAD in dialysis patients, ${ }^{3}$ the following are stated:

(1) All patients at the initiation of dialysis regardless of symptoms require assessment for cardiovascular disease as well as screening for both traditional and non-traditional cardiovascular risk factors.

(2) "Automatic' CAD evaluation with stress imaging is currently not recommended for all dialysis patients.

(3) Stress imaging is appropriate in selected high-risk dialysis patients for risk stratification even in patients who are not renal transplant candidates (high-risk defined as Framingham risk score of $>20 \%$ ).

From the Division of Cardiovascular Diseases, ${ }^{\text {a }}$ Henry Ford Hospital, Detroit, MI; and Division of Cardiovascular Diseases, ${ }^{\text {b }}$ William Beaumont Hospital, Royal Oak, MI.

Reprint requests: Abdul Hakeem, MD, Division of Cardiovascular Diseases, William Beaumont Hospital, 13 Mile Road, Royal Oak, MI; ahakeem@gmail.com.

J Nucl Cardiol 2012;19:410-1.

$1071-3581 / \$ 34.00$

Copyright (c) 2012 American Society of Nuclear Cardiology.

doi:10.1007/s12350-012-9524-y
Several aspects that are unique to ESRD make risk stratification challenging. ${ }^{4}$ First, this is a high-risk cohort and further risk discrimination (establishing incremental value over baseline risk) in such a cohort with high pretest likelihood is always challenging. Second, endothelial dysfunction, left ventricular hypertrophy $(\mathrm{LVH})$, and volume and pressure overload likely affect the performance of established imaging modalities in quantification of disease burden as well as subsequent prognostication. An inability to exercise attributed to multiple comorbidities limits access to the most extensively validated prognostic information which is total exercise capacity; hence, necessitating use of pharmacological agents, which may be suboptimal in ESRD patients due to an incomplete vasodilator response because of significant endothelial dysfunction. The presence of severe $\mathrm{LVH}$ also masks the presence of small defects due to limited spatial resolution of myocardial perfusion imaging (MPI). Similarly, dobutamine stress echo (DSE) is limited by significant false negative rates related to limitations with the use of dobutamine and lower sensitivity to detect wall motion abnormalities in severe LVH. This hypertensive response also compromises diagnostic utility secondary to transient LV dysfunction and wall motion abnormality in the absence of CAD. Despite the limitations associated with each form of pharmacologic stress testing, DSE and SPECT remain valuable techniques for diagnosis and effective risk stratification of patients with CKD. A large pool of evidence has been generated over the years that validate the value of noninvasive risk stratification in this population. ${ }^{4}$

In the current issue of $\mathrm{JNC}, \mathrm{Kim}$ et $\mathrm{al}^{5}$ present the results of a well-designed single center prospective study from Korea. Using clinical and echo parameters, 215 patients at the start of dialysis were categorized into low risk and high clinical risk. High-risk patients were further "screened"' using MPI. During an average follow-up of 4 years, $31 \%$ in the high-risk group and $4 \%$ in the low-risk group had cardiac events. The overall rate of cardiac events per person-year of follow-up was $15 \%$ in the high-risk group with perfusion defects, $4.5 \%$ in the high-risk group without perfusion defects, and $1.2 \%$ in the low-risk group. On multivariable analysis, DM, perfusion defect, and left ventricular ejection fraction (LVEF) were independent predictors of adverse events. The presence of perfusion defects effectively 
characterized as high-risk patients; those with perfusion defects had a 3.3-fold higher risk of events compared to those without defects. The authors further demonstrated the "incremental" value of MPI "screening", in highrisk patients by demonstrating a significant increase in global chi-square score with the addition of MPI data to the baseline + echo model. Similarly, on receiver operator curve analysis, a significant increase in the C-Statistic was demonstrated with the addition of MPI in comparison to base line alone and baseline + echo model.

Baseline risk stratification using clinical variables is very effective, even in a high-risk cohort such as this. Patients with a normal LVEF, $<2$ risk factors and no history of long-term diabetes, as demonstrated in this study, identifies a low-risk group with a very low event rate $(1.2 \%$ cardiac event rate per person year of followup) which probably does not need further testing unless clinically indicated. For those categorized as "high risk' on the initial screen, further effective discrimination is provided by MPI data. This is in keeping with the most recent criteria of appropriate use. ${ }^{6}$

Earlier studies have established the prognostic value of MPI in patients with ESRD. ${ }^{4} \mathrm{~A}$ meta-analysis by Rabbat et al evaluated the prognostic utility of stress MPI for predicting MI and cardiac death in patients with ESRD assessed for kidney or kidney-pancreas transplantation. ${ }^{7}$ The authors included 12 studies, 8 of which evaluated thallium scintigraphy and 4 evaluated DSE. They demonstrated that patients with evidence of inducible ischemia had a sixfold risk of MI and a fourfold risk of cardiac death, whereas fixed defects were only predictive of cardiac death (RR 4.7) and not MI. Multiple studies subsequent to this meta-analysis have been published that have established the prognostic utility of MPI in ESRD patients. ${ }^{4}$ Venkataraman et $\mathrm{al}^{8}$ showed that the larger the defect size on vasodilator stress MPI, the worse the prognosis for patients on hemodialysis. Perfusion defects on MPI provided better prognostication compared to the severity of angiographic coronary stenosis. In multivariate analysis, abnormal MPI (low LVEF or abnormal perfusion) and diabetes alone were independent predictors of death, whereas number of narrowed arteries on coronary angiography was not as predictive. ${ }^{8}$

Although patients who received only medical treatment appeared to have a higher rate of cardiac events, revascularization therapy did not improve the cardiac eventfree survival rate. This finding must be interpreted with caution as this was a small sample size, non-randomized and extent of revascularization (complete vs. incomplete) was not reported. Furthermore, $36 \%$ patients with reversible perfusion defects did not undergo diagnostic catheterization. Along the same line, medical therapy was suboptimal with $<60 \%$ patients being on aspirin, beta blockers, and statin. There remains clinical equipoise regarding medical management or invasive strategy for patients with ESRD and perfusion abnormalities. Additionally, whether repeat stress testing should be performed in patients with ESRD, especially those with normal MPI, remains to be seen.

Most clinical trials have by convention excluded patients with CKD and more importantly patients with ESRD. Prospective well-designed multicenter trials are urgently needed to examine the evaluation and management strategies for cardiovascular risk in dialysis patients.

\section{References}

1. AHA Statistical Update: Heart Disease and Stroke Statistics-2011 Update. Circulation 2011;123:e18-e209.

2. de Jager DJ, Grootendorst DC, Jager KJ, et al. Cardiovascular and noncardiovascular mortality among patients starting dialysis. JAMA 2009;302:1782-9.

3. http://www.kidney.org/professionals/kdoqi/guidelines_cvd/guide2. htm. Accessed 1/1/2012.

4. Hakeem A, Bhatti S, Trevino AR, Samad Z, Chang SM. Noninvasive risk assessment in patients with chronic kidney disease. J Nucl Cardiol 2011;18:472-85.

5. Kim J-K, Kim SG, Kim HJ, Song YR. Cardiac risk assessment by gated single-photon emission computed tomography in asymptomatic end-state renal disease patients at the start of dialysis. J Nucl Cardiol 2011. doi:10.1007/s12350-011-9497-2.

6. Hendel RC, Berman DS, Di Carli MF, et al. ACCF/ASNC/ACR/ AHA/ASE/SCCT/SCMR/SNM 2009 appropriate use criteria for cardiac radionuclide imaging. J Am Coll Cardiol 2009;53:2201-29.

7. Rabbat CG, Treleaven DJ, Russell JD, Ludwin D, Cook DJ. Prognostic value of myocardial perfusion studies in patients with end-stage renal disease assessed for kidney or kidney-pancreas transplantation: A meta-analysis. J Am Soc Nephrol 2003;14:431-9.

8. Venkataraman R, Hage FG, Dorfman T, Heo J, Aquel RA, de Mattos AM, et al. Role of myocardial perfusion imaging in patients with end-stage renal disease undergoing coronary angiography. Am J Cardiol 2008;102:1451-6. 